\title{
The quantitative genetic basis of polyandry in the parasitoid wasp, Nasonia vitripennis
}

\author{
DM Shuker, AJ Phillimore, MN Burton-Chellew, SE Hodge and SA West \\ Institute of Evolutionary Biology, School of Biological Sciences, University of Edinburgh, Edinburgh, UK
}

Understanding the evolution of female multiple mating (polyandry) is crucial for understanding sexual selection and sexual conflict. Despite this interest, little is known about its genetic basis or whether genetics influences the evolutionary origin or maintenance of polyandry. Here, we explore the quantitative genetic basis of polyandry in the parasitoid wasp Nasonia vitripennis, a species in which female remating has been observed to evolve in the laboratory. We performed a quantitative genetic experiment on a recently collected population of wasps. We found low heritabilities of female polyandry (re-mating frequency after 18h), low heritability of courtship duration and a slightly higher heritability of copulation duration. However, the coefficients of additive genetic variance for these traits were all reasonably large $\left(C V_{\mathrm{A}}>7.0\right)$. We also found considerable dam effects for all traits after controlling for common environment, suggesting either dominance or maternal effects. Our work adds to the evidence that nonadditive genetic effects may influence the evolution of mating behaviour in Nasonia vitripennis, and the evolution of polyandry more generally. Heredity (2007) 98, 69-73. doi:10.1038/sj.hdy.6800897; published online 20 September 2006

Keywords: additive genetic variance; heritability; Hymenoptera; polyandry; sexual selection; sexual conflict

\section{Introduction}

The evolution of female multiple mating is a puzzle in species in which males provide little or no resources apart from sperm (Ridley, 1988; Andersson, 1994). In many cases female fertility is maximized by a limited number of copulations (Ârnqvist and Nilsson, 2000). However, the emerging picture from a wide range of species is that females mate more frequently than necessary for full fertility, risking elevated costs associated with numerous matings (Daly, 1978; Chapman et al., 1995; Shuker et al., 2006). Explanations for female multiple mating with different partners (polyandry) have concentrated on either the potential genetic benefits that become available to females by sampling multiple males via copulation (Andersson, 1994; Eberhard, 1996; Jennions and Petrie, 2000; Radwan, 2003; Simmons, 2005), or on the fact that the observed female mating rate might represent a sexual conflict of interests (Parker, 1979; Thornhill and Alcock, 1983; Brown et al., 1997; Rice, 1998; Chapman et al., 2003).

Understanding the evolution of polyandry is therefore important for understanding the role of females in sexual selection, in particular female choice, and for the life history consequences of reproduction. It is also important for understanding the scope of sexual conflict, since polyandry may arise as a consequence of sexual conflict over mating (e.g. Rowe et al., 1994; Shuker and Day, 2001;

Correspondence: Dr DM Shuker, Institute of Evolutionary Biology, School of Biological Sciences, University of Edinburgh, West Mains Road, Edinburgh, EH9 3JT, UK.

E-mail:david.shuker@ed.ac.uk

Received 13 June 2006; revised 24 July 2006; accepted 16 August 2006; published online 20 September 2006
Shuker and Day, 2002), but may also be a cause of sexual conflict by forcing males into sperm competition and enabling females to make cryptic mate choices (Parker, 1970; Birkhead and Moller, 1998; Simmons, 2001). Despite the interest in the evolution and maintenance of polyandry, its genetic basis has received less attention. While the genetic basis of mating behaviour has been considered in detail in Drosophila melanogaster (e.g. Hall, 1994; Sokolowski, 2001; Mackay et al., 2005), it is only relatively recently that polyandry has been examined genetically in a wider variety of insects (Orthoptera: Solymar and Cade, 1990; Lepidoptera: Torres-Vila et al. 2001; Wedell, 2001; Torres-Vila et al., 2002; Wedell et al., 2002; Coleoptera: Harano and Miyatake, 2005; Hymenoptera: Kraus et al., 2005).

We have recently observed polyandry evolving in laboratory populations of the parasitoid wasp, Nasonia vitripennis (Burton-Chellew et al., unpublished data). Freshly collected females from the wild do not readily re-mate (Van den Assem et al., 1980; Van den Assem and Jachmann, 1999), although they are not strictly monandrous. However, the propensity of females to re-mate evolves repeatedly in the laboratory, increasing over time (Van den Assem and Jachmann, 1999). Estimates of levels of polyandry in the wild have not been obtained, although traditionally females have been thought to mate only once, immediately on emergence from their host before dispersing to find oviposition sites (Whiting, 1967). The reasons for this increase in polyandry in $N$. vitripennis are currently not clear, although the changes in polyandry are associated with changes in female mating behaviour (Burton-Chellew et al., unpublished data).

Here, we consider the quantitative genetic basis of polyandry in a recently collected population 
of $N$. vitripennis, measuring polyandry as the likelihood of re-mating after $18 \mathrm{~h}$. We show that female re-mating behaviour has a low heritability but is influenced by large, significant maternal or nonadditive genetic effects.

\section{Materials and methods}

\section{Mating behaviour of $N$. vitripennis}

N. vitripennis (Hymenoptera: Pteromalidae) is a gregarious parasitoid of large dipteran pupae (e.g. Calliphoridae and Sarcophagidae: Whiting, 1967). Courtship involves a stereotyped series of events (Barass, 1960). Following contact, the male mounts the female and positions his head over her antennae. Courtship begins with a series of head nods, which continue in a rhythmic display consisting of approx 4-7 nods per series, with a short pause between each series. The first head nod of each series is associated with the release of a mandibular pheromone (Van den Assem et al., 1980). Virgin females generally display receptivity after a short bout of courtship, drawing their antennae forward and arching their abdomens. The male will back up, establish genital contact, and inseminate the female. Copulation typically lasts around 10-15s after which the male moves back to perform a short bout of postcopulatory courtship before dismounting.

\section{The study population}

The laboratory population was generated from founding individuals collected from three different nest boxes from Hoge Veluwe forest, the Netherlands in June 2004. Reciprocal crosses between males and females from each nest box generated nine crosses, replicated 15 times. These 135 mated females were distributed between three stock tubes of hosts (fly pupae). Ten days postoviposition parasitized hosts were removed from and then redistributed back into the tubes at random. Three generations of random mating and host mixing ensued, with approximately 150 females founding each generation, producing an outbred population hereafter referred to as HV04. All cultures were maintained at $25^{\circ} \mathrm{C}$ with a $18: 6$ light:dark cycle. Under these conditions the generation time is approximately 14 days.

\section{Quantitative genetic experiment}

We performed a standard split-family sib analysis (Falconer and Mackay, 1996; Roff, 1997; Lynch and Walsh, 1998). Virgin wasps were collected from the population culture 2 days before emergence. A large number of parasitized hosts were opened and virgin male and female wasp pupae were mixed with a fine paintbrush. Full-sib/half-sib families were created by making mating groups of one male pupa (sire) with five female pupae (dams). Following emergence, we allowed females to mate for 2 days after which we placed each female individually into a tube with two host pupae, so that larval environments were split within full-sib families. After 2 days of oviposition we removed the female and placed the hosts in separate tubes. We randomly distributed these tubes across test tube racks, so that hosts from the same families were distributed across racks and shelves in the incubator. Additional virgin females from the population culture were maintained separately from the experimental families in order to generate virgin males for mating trials. Approximately 20 females were placed into a culture tube with around 40 hosts. This was carried out every 3 days generating a continuous supply of inexperienced experimental males.

Two days before offspring emergence, we opened each host and collected five virgin females, yielding 10 experimental daughters per full-sib family. The day following emergence we fed the females with honey solution-soaked filter paper. Before both the primary and secondary mating trials we removed all experimental females and males from the incubator at least $30 \mathrm{~min}$ before the experiment took place to allow acclimatization. To standardize light conditions, both primary and secondary matings were observed on a white board illuminated by a Euromex Fiber Optic light source EK-1 at setting eight.

\section{Mating trials}

To test female polyandry, each female was given two mating trials, separated by approximately $18 \mathrm{~h}$. We performed all primary matings between 1700 and 2000 hours at ambient temperature (median temperature $23^{\circ} \mathrm{C}$ ). A female was extracted from the test tube and placed in an observation vial with a virgin male already present. We recorded the time in seconds to the onset of courtship, the onset of copulation and the end of copulation. Postcopulatory courtship was allowed after which we removed the female and placed her in a solitary test tube. Any female who had not been courted after $5 \mathrm{~min}$ was discarded. From these data we obtained the courtship duration and copulation duration for virgin females. As expected, acceptance rates of the virgin females were uniformly high $(97.6 \%$ of females mated in the first mating trial).

The re-mating assay took place approximately $18 \mathrm{~h}$ later (again median temperature was $23^{\circ} \mathrm{C}$ ). Each female was observed for $10 \mathrm{~min}$ or until copulation. For each female we recorded receptivity at first courtship $\left(R_{1}\right)$ and receptivity within $10 \mathrm{~min}\left(R_{10}\right)$. Any females engaged in courtship at $10 \mathrm{~min}$ were allowed to finish courtship and commence copulation if they accepted the male. Any females who had not been courted within the $10 \mathrm{~min}$ were not included in the results.

To ensure that the observed mating events did involve sperm transfer, we repeated the mating trials with 46 virgin males and females from the stock culture, giving each female a tube containing three hosts after copulation. Forty-one of the females laid eggs, and all of these females produced daughters, confirming sperm transfer.

\section{Quantitative genetic analysis}

In total, 1005 individuals, representing 24 half-sib, and 106 full-sib families were analysed for our four mating traits (courtship duration, copulation duration, $R_{1}$ and $R_{10}$ ). We obtained variance components from linear mixed models with REML in S-Plus 7 (Insightful Corporation, Seattle, WA, USA). For haplodiploids (Liu and Smith, 2000), the sire variance component consists of additive genetic effects (such that $\sigma_{\text {sire }}^{2}=V_{\mathrm{A}} / 2$ ) and the dam within sire component consists of additive, dominance and common environmental components $\left(\sigma_{\mathrm{dam}(\text { sire })}^{2}=V_{\mathrm{A}} / 4+V_{\mathrm{D}} / 2+V_{\mathrm{EC}}\right)$. The two measures of polyandry $\left(R_{1}\right.$ and $\left.R_{10}\right)$ were modelled as threshold characters, assuming continuous variation (liability) on 
an underlying scale (Falconer and Mackay, 1996; Roff, 1997; Lynch and Walsh, 1998). The heritability was measured first on the observed $(0,1)$ scale and then converted to an estimate on the underlying scale:

$$
h^{2}=h_{\text {obs }}^{2}[p(1-p)] / z^{2}
$$

where $h_{\mathrm{obs}}^{2}$ is the heritability on the observed scale, $p$ is the proportion of females re-mating, and $z$ is the ordinate on the standardized normal curve in the underlying scale corresponding to $p$, calculated as

$$
z=\left[\mathrm{e}^{\left(-0.5 X^{2}\right)}\right] / \sqrt{ } 2 \pi
$$

where $X=[\operatorname{sign}(0.5-p)][1.238 c(1+0.0262 c)]$, and $c=$ $\sqrt{ }-\ln [4 p(1-p)]$.

The approximate standard error is obtained from:

$$
\text { s.e. }\left(h^{2}\right)=\text { s.e. }\left(h_{0,1}^{2}\right)[p(1-p)] / z^{2}
$$

where s.e. $\left(h_{0,1}^{2}\right)$ is the standard error on the observed scale. The coefficient of additive genetic variation $\left(C V_{\mathrm{A}}\right)$ was calculated as $C V_{\mathrm{A}}=100 \sqrt{V_{\mathrm{A}} /} \bar{x}$ (on the observed scale for the polyandry measures) following Houle (1992).

Our heritability estimates for all traits were low, making interpretation of genetic correlations problematic given the necessarily large standard errors (Roff, 1997; Lynch and Walsh, 1998). We therefore only present the phenotypic correlation between courtship duration and copulation duration, and use logistic regression to explore the effect of copulation duration and courtship duration in the first mating trial on the likelihood of re-mating (as both $R_{1}$ and $R_{10}$ ).

\section{Results}

\section{Descriptive statistics}

The mean courtship duration was $10.6 \pm 0.3 \mathrm{~s}$ and the mean copulation duration was $14.9 \pm 0.1 \mathrm{~s}$ (both $N=1005)$. The frequency of re-mating in the second mating trial in response to the first courtship was $R_{1}=0.46$, and within $10 \mathrm{~min}$ was $R_{10}=0.70$.

\section{Quantitative genetic estimates}

The genetics of female re-mating were dominated by dam effects (Table 1). The heritabilities for all traits were low $\left(h^{2}<0.15\right)$, with only copulation duration having significant heritability, although the heritability of $R_{1}$ is close to significance. There were significant dam effects for all traits, while common environment variance components were small (Table 1). The coefficients of additive genetic variation, however, suggest there is a reasonable amount of additive genetic variation swamped by the nonadditive variation for all four traits $\left(C V_{\mathrm{A}}=7.01-19.98\right.$; Table 2).

\section{Phenotypic relationships between traits}

Females that were courted for longer also tended to copulate for longer. There was a weak but highly significant positive phenotypic correlation between courtship duration and copulation duration; however, the correlation explained very little of the variation in the data $\left(r_{1003}=0.104, P=0.0009, R^{2}=1.1 \%\right)$. Aspects of the first mating trial had at best only weak influence on female re-mating. There were weak negative relationships between courtship or copulation duration and
Table 1 Variance components and estimates of the heritability through the sire and dam for the four traits with their standard errors $(N=1005$ individuals, from 106 dams and 24 sires)

\begin{tabular}{lcccc}
\hline & Sire & Dam & Host & Residual \\
\hline Variance components & & & & \\
$R_{1}$ & 0.00829 & 0.02183 & 0.00464 & 0.21442 \\
$R_{10}$ & 0.00452 & 0.02496 & 0.00001 & 0.18154 \\
Courtship duration & 1.22871 & 4.59652 & 2.97146 & 76.00258 \\
Copulation duration & 0.55618 & 0.99172 & 0.27165 & 5.57319 \\
& & & & \\
Heritability & Sire & s.e. & Dam & s.e. \\
$R_{1}{ }^{a}$ & 0.105 & 0.060 & $\mathbf{0 . 5 5 3}$ & 0.168 \\
$R_{10}{ }^{2}$ & 0.074 & 0.052 & $\mathbf{0 . 8 2 0}$ & 0.206 \\
Courtship duration & 0.029 & 0.026 & $\mathbf{0 . 2 1 7}$ & 0.093 \\
Copulation duration & $\mathbf{0 . 1 5 0}$ & 0.063 & $\mathbf{0 . 5 3 7}$ & 0.126 \\
\hline
\end{tabular}

Heritabilities significant at the 5\% level are highlighted in bold. ${ }^{a}$ Heritabilities presented on the underlying scale.

Table 2 Coefficients of additive genetic variation $\left(C V_{\mathrm{A}}\right.$ : following Houle, 1992) for the four traits

\begin{tabular}{ccccc}
\hline Trait & $R_{1}$ & $R_{10}$ & Courtship duration & Copulation duration \\
\hline $\mathrm{CV}_{\mathrm{A}}$ & 19.98 & 9.61 & 7.01 & 7.43
\end{tabular}

$C V_{\mathrm{A}} \mathrm{s}$ calculated using the sire estimate of the additive genetic variance.

Table 3 Phenotypic relationships between female polyandry $\left(R_{1}\right.$ and $R_{10}$ ) and courtship duration and copulation duration analysed via logistic regression

\begin{tabular}{llcccc}
\hline Polyandry & Behaviour & $\beta$ (s.e.) & $\chi^{2}$ & $\mathrm{P}$ & $\mathrm{R}^{2}(\%)$ \\
\hline$R_{1}$ & Courtship & $-0.021(0.008)$ & 8.06 & 0.005 & 0.6 \\
$R_{1}$ & Copulation & $-0.006(0.023)$ & 0.06 & 0.81 & 0.0 \\
$R_{10}$ & Courtship & $-0.015(0.007)$ & 4.09 & 0.04 & 0.3 \\
$R_{10}$ & Copulation & $-0.056(0.025)$ & 5.09 & 0.02 & 0.4 \\
\hline
\end{tabular}

$\beta$ (s.e.) is the logistic regression coefficient (standard error). Significance tested via likelihood $\chi^{2}$-tests.

$R_{1}$ or $R_{10}$, all of which explained less than $1 \%$ of the variation in likelihood of re-mating (Table 3). The most convincing relationship was that between courtship duration and receptivity at first courtship (logistic regression coefficient: $\beta=-0.021 \pm 0.008$, $\chi^{2}=8.06, P=0.005, R^{2}=0.6 \%$ ).

\section{Discussion}

The genetic basis of polyandry in our experiment was dominated by nonadditive genetic or maternal effects. Despite nontrivial coefficients of additive genetic variation, heritabilities of female re-mating and copulation and courtship duration were therefore low. Behavioural traits typically exhibit low heritabilities (Mousseau and Roff, 1987; Merila and Sheldon, 2000; Boake et al., 2002) and this is often attributed to the inherent variability of behaviours, especially when individuals are interacting. Here, we have both residual environmental variation (after experimentally and statistically limiting common environmental effects) and substantial nonadditive genetic effects (Table 1). These latter effects could include 
both dominance and/or some other maternally inherited variation (Meffert et al., 2002). While we have no specific estimates for the dominance variance component for these female mating behaviours, they would have to be larger than average for behavioural traits to explain all the nonadditive variation (e.g. Crnokrak and Roff, 1995; Roff, 1997; Stirling et al., 2002), suggesting that the fullsib variances are influenced by some maternal effects as well. However, dominance effects have recently been shown to influence variation in polyandry in the beetle Callosobruchus chinensis (Harano and Miyatake, 2005) and aspects of courtship behaviour in houseflies (Meffert and Hagenbuch, 2005). Dominance may also play a part in maintaining heritable variation in re-mating rate in the wild (Crnokrak and Roff, 1995).

How might a maternal effect arise in N. vitripennis? Even though there is no parental care, females may influence variation in offspring phenotypes via their energetic investment in eggs or by influencing the degree of larval competition within a host via clutch size (Godfray, 1994). Our split-family design should have minimized the latter, but we do not know how females vary in their patterns of egg investment (although recent work suggests that egg size variation does not have a large effect on larval performance in $N$. vitripennis: Lalonde, 2005). In addition, female venom mobilizes host lipid reserves into the host's haemolymph (Rivers, 2004), providing another possible source of maternal effect. Other nonadditive genetic effects influencing Nasonia mating behaviour have also been uncovered in the context of the courtship behaviour of interspecific male hybrids (Beukeboom and Van den Assem, 2001; Beukeboom and Van den Assem, 2002). In a series of experiments, hybrid males tended to resemble their maternal grandfathers in terms of courtship phenotype (since males are haploid, they do not have paternal grandfathers, only maternal grandfathers). While the mechanistic basis for this effect is still unclear, one possibility is that grand-paternally imprinted genes influence grand-offspring behaviour. It remains to be seen whether any of our nonadditive effects could have a similar basis (see also Mills and Moore, 2004).

As with all heritability studies, specific estimates for polyandry will vary with the environment and also the explicit experimental protocol (Falconer and Mackay, 1996). However, our results are consistent with other recent work, despite the fact that most studies have not specifically controlled for nonadditive effects. For example, broad-sense heritabilities in excess of 0.4 have been found in crickets, butterflies and moths (Solymar and Cade 1990; Torres-Vila et al., 2001, 2002; Wedell et al., 2002) and our estimates of polyandry heritability via dams are likewise large and significant. Our data also confirm that species deemed to be for the most part monandrous can have segregating additive genetic variation in re-mating rate (i.e. the moth Lobesia botrana; Torres-Vila et al., 2002). While the evolutionary changes in female re-mating frequency observed in the laboratory attest to this variation, our results suggest that nonadditive effects may have contributed to this response and influenced the rate of evolutionary response (Burton-Chellew et al., unpublished data).

Phenotypically the four mating traits we scored were fairly independent of each other, with only weak correlations between them. While there seems to be a general trend for females that took longer to court or copulate to be less likely to re-mate, it does not explain much of the variation in female polyandry. Such as it is, the trend may reflect overall female receptivity. However, there could also be a mechanistic or sexual selection component. For example, female re-mating could depend on some aspect of first or second male quality, such as the volume of sperm transferred. We have not explicitly considered the role of males here, but sperm can be an important limiting factor on female fitness in parasitoid wasps (e.g. Godfray, 1994; Hardy et al., 1998; West et al., 1998; West et al., 1997). Males may vary in the numbers of sperm they transfer (Henter, 2004; Shuker et al., 2006) and males of some species even continue to copulate when they are sperm-depleted (Damiens and Boivin, 2006). We have some evidence that female remating per se is mechanistically independent of male mating partner (Burton-Chellew et al., unpublished data), but in broader terms we may expect aspects of male and female mating behaviour to coevolve, with female multiple mating becoming more likely if males are selected to mate beyond their ability to successfully inseminate females. We are currently exploring these aspects of male mating competitiveness in Nasonia and how they influence female behaviour and reproductive allocation.

\section{Acknowledgements}

We are very grateful to the Natural Environment Research Council, the Biotechnology and Biological Sciences Research Council and the Royal Society for funding. Dr A Hoyahoy provided useful comments on an earlier version of the manuscript, and Dr Alan Gemmill showed us evollusion.

\section{References}

Andersson M (1994). Sexual Selection. Princeton University Press: Princeton.

Arnqvist G, Nilsson T (2000). The evolution of polyandry: multiple mating and female fitness in insects. Anim Behav 60: 145-164.

Barass R (1960). The courtship behaviour of Mormoniella vitripennis walk (Hymenoptera, Pteromalidae). Behaviour 15: 185-209.

Beukeboom LW, Van den Assem J (2001). Courtship and mating behaviour of interspecific Nasonia hybrids (Hymenoptera, Pteromalidae): a grandfather effect. Behav Genet 31: 167-177.

Beukeboom LW, Van den Assem J (2002). Courtship displays of introgressed, interspecific hybrid Nasonia males: further investigations into the 'grandfather effect'. Behaviour 139: 1029-1042.

Birkhead TR, Moller AP (1998). Sperm Competition and Sexual Selection. Academic Press: San Diego.

Boake CRB, Arnold SJ, Breden F, Meffert LM, Ritchie MG, Taylor BJ et al. (2002). Genetic tools for studying adaptation and the evolution of behavior. Amer Nat 160: S143-S159.

Brown WD, Crespi BJ, Choe JC (1997). Sexual conflict and the evolution of mating systems. In: Choe JC, Crespi BJ (eds). The Evolution of Mating Systems in Insects and Arachnids. Cambridge University Press: Cambridge. pp 352-377.

Chapman T, Arnqvist G, Bangham J, Rowe L (2003). Sexual conflict. Trends Ecol Evol 18: 41-47.

Chapman T, Liddle LF, Kalb JM, Wolfner MF, Partridge L (1995). Cost of mating in Drosophila melanogaster females is mediated by male accessory gland products. Nature 373: 241-244. 
Crnokrak P, Roff DA (1995). Dominance variance - associations with selection and fitness. Heredity 75: 530-540.

Daly M (1978). Cost of mating. Amer Nat 112: 771-774.

Damiens D, Boivin G (2006). Why do sperm-depleted parasitoid males continue to mate? Behav Ecol 17: 138-143.

Eberhard WG (1996). Female Control: Sexual Selection by Cryptic Female Choice. Princeton University Press: Princeton.

Falconer DS, Mackay TFC (1996). Introduction to Quantitative Genetics, 4th edn. Longman: Harlow.

Godfray HCJ (1994). Parasitoids Behavioural and Evolutionary Ecology. Princeton University Press: Princeton.

Hall JC (1994). The mating of a fly. Science 264: 1702-1714.

Harano T, Miyatake T (2005). Heritable variation in polyandry in Callosobruchus chinensis. Anim Behav 70: 299-304.

Hardy ICW, Dijkstra LJ, Gillis JEM, Luft PA (1998). Patterns of sex ratio, virginity and developmental mortality in gregarious parasitoids. Biol J Linn Soc 64: 239-270.

Henter HJ (2004). Constrained sex allocation in a parasitoid due to variation in male quality. J Evol Biol 17: 886-896.

Houle D (1992). Comparing evolvability and variability of quantitative traits. Genetics 130: 195-204.

Jennions MD, Petrie M (2000). Why do females mate multiply? A review of the genetic benefits. Biol Rev 75: 21-64.

Kraus FB, Neumann P, Moritz RFA (2005). Genetic variance of mating frequency in the honeybee (Apis mellifera L.). Insect Soc 52: $1-5$.

Lalonde RG (2005). Egg size variation does not affect offspring performance under intraspecific competition in Nasonia vitripennis, a gregarious parasitoid. I Anim Ecol 74: 630-635.

Liu FH, Smith SM (2000). Estimating quantitative genetic parameters in haplodiploid organisms. Heredity 85: 373-382.

Lynch M, Walsh B (1998). Genetics and Analysis of Quantitative Traits. Sinauer Associates: Sunderland, MA.

Mackay TFC, Heinsohn SL, Lyman RF, Moehring AJ, Morgan TJ, Rollmann SM (2005). Genetics and genomics of Drosophila mating behavior. Proc Natl Acad Sci USA 102: 6622-6629.

Meffert LM, Hagenbuch KL (2005). The genetic architecture of house fly mating behavior. Curr Topics Dev Biol 66: 189-213.

Meffert LM, Hicks SK, Regan JL (2002). Nonadditive genetic effects in animal behavior. Amer Nat 160: S198-S213.

Merila J, Sheldon BC (2000). Lifetime reproductive success and heritability in nature. Amer Nat 155: 301-310.

Mills W, Moore T (2004). Polyandry, life-history trade-offs and the evolution of imprinting at Mendelian loci. Genetics 168: 2317-2327.

Mousseau TA, Roff DA (1987). Natural-selection and the heritability of fitness components. Heredity 59: 181-197.

Parker GA (1970). Sperm competition and its evolutionary consequences in the insects. Biol Rev 45: 525-567.

Parker GA (1979). Sexual selection and sexual conflict. In: Blum MS, Blum NB (eds). Sexual Selection and Reproductive Competition in Insects. Academic Press: New York. pp 123-166.

Radwan J (2003). Male age, germline mutations and the benefits of polyandry. Ecol Lett 6: 581-586.

Rice WR (1998). Intergenomic conflict, interlocus antagonistic coevolution, and the evolution of reproductive isolation. In: Howard DJ, Berlocher SH (eds). Endless Forms. Oxford University Press: Oxford. pp 261-270.

Ridley M (1988). Mating frequency and fecundity in insects. Biol Rev 63: 509-549.

Rivers DB (2004). Evaluation of host responses to envenomation as a means to assess ectoparasitic pteromalid wasp's potential for controlling manure-breeding flies. Biol Control 30: $181-192$.

Roff DA (1997). Evolutionary Quantitative Genetics. Chapman \& Hall: New York.

Rowe L, Arnqvist G, Sih A, Krupa J (1994). Sexual conflict and the evolutionary ecology of mating patterns - water striders as a model system. Trends Ecol Evol 9: 289-293.

Shuker DM, Ballantyne GA, Wedell N (2006). Variation in the cost to females of the sexual conflict over mating in the seed bug Lygaeus equestris (Hemiptera: Lygaeidae). Anim Behav 72: 313-321.

Shuker DM, Day TH (2001). The repeatability of a sexual conflict over mating. Anim Behav 61: 755-762.

Shuker DM, Day TH (2002). Mate sampling and the sexual conflict over mating in seaweed flies. Behav Ecol 13: 83-86.

Shuker DM, Sykes EM, Browning LE, Beukeboom LW, West SA (2006). Male influence on sex allocation in the parasitoid wasp Nasonia vitripennis. Behav Ecol Sociobiol 59: 829-835.

Simmons LW (2001). Sperm Competition and its Evolutionary Consequences in the Insects. Princeton University Press: Princeton.

Simmons LW (2005). The evolution of polyandry: sperm competition, sperm selection, and offspring viability. Annu Rev Ecol Evol Syst 36: 125-146.

Sokolowski MB (2001). Drosophila: genetics meets behaviour. Nature Rev Genet 2: 879-890.

Solymar BD, Cade WH (1990). Heritable variation for female mating frequency in field crickets, Gryllus integer. Behav Ecol Sociobiol 26: 73-76.

Stirling DG, Reale D, Roff DA (2002). Selection, structure and the heritability of behaviour. J Evol Biol 15: 277-289.

Thornhill R, Alcock J (1983). The Evolution of Insect Mating Systems. Harvard University Press: Harvard.

Torres-Vila LM, Gragera J, Rodriguez-Molina MC, Stockel J (2002). Heritable variation for female remating in Lobesia botrana, a usually monandrous moth. Anim Behav 64: 899-907.

Torres-Vila LM, Rodriguez-Molina MC, Gragera J, Bielza-Lino P (2001). Polyandry in Lepidoptera: a heritable trait in Spodoptera exigua Hubner. Heredity 86: 177-183.

Van den Assem J, Jachmann F (1999). Changes in male perseverance in courtship and female readiness to mate in a strain of the parasitic wasp Nasonia vitripennis over a period of 20+years. Neth J Zool 49: 125-137.

Van den Assem J, Jachmann F, Simbolotti P (1980). Courtship behaviour of Nasonia vitripennis (Hym. Pteromalidae): some qualitative, experimental evidence for the role of pheromones. Behaviour 75: 301-307.

Wedell N (2001). Female remating in butterflies: interaction between female genotype and nonfertile sperm. J Evol Biol 14: 746-754.

Wedell N, Wiklund C, Cook PA (2002). Monandry and polyandry as alternative lifestyles in a butterfly. Behav Ecol 13: $450-455$.

West SA, Compton SG, Vincent SL, Herre EA, Cook JM (1998). Virginity in haplodiploid populations: a comparison of estimation methods. Ecol Entomol 23: 207-210.

West SA, Herre EA, Compton SG, Godfray HCJ, Cook JM (1997). A comparative study of virginity in fig wasps. Anim Behav 54: 437-450.

Whiting AR (1967). The biology of the parasitic wasp Mormoniella vitripennis. Q Rev Biol 42: 333-406. 